\title{
Thinking clearly about postoperative delirium and perioperative medications: how concerned should we be?
}

\author{
Nabil Elkassabany, MD · Stanley Muravchick, MD, PhD • \\ Lee A. Fleisher, MD
}

Published online: 27 August 2009

(C) Canadian Anesthesiologists' Society 2009

Delirium has been described since Hippocrates' time. In 1837, Sir Charles Dickens wrote the following detailed description of the syndrome in The Pickwick Papers-The Stroller's Tale, "The mood in this account is also a mixture of frivolous buffoonery and abject terror. Illusions and hallucinations abound". 1 Since then, the understanding of this condition has been evolving. As anesthesiologists, we and other physicians involved in patient care in the perioperative period are concerned about postoperative delirium (POD). In the current issue of the Journal, Katznelson et $a .^{2}$ have added to the literature by performing a retrospective chart review to determine the association between the perioperative use of beta-blockers and statins and the frequency of postoperative delirium in vascular surgery patients. They report a twofold increase in the odds ratio of postoperative delirium in patients receiving preoperative beta-blockers (odds ratio 2.06 with a $95 \%$ confidence interval [1.18-3.6]) and a 44\% reduction in the odds ratio of postoperative delirium in patients receiving statins.

Delirium is a final common pathway for a wide range of insults that compromise a vulnerable nervous system with limited functional reserve. It is clinically defined as an acute confusional state characterized by disorientation, a disturbed sleep-wake cycle, memory impairment, perceptual disturbances, and altered psychomotor activity. Because of these disparate and subjective symptoms, it is not surprising

N. Elkassabany, MD (ه) S. Muravchick, MD, PhD .

L. A. Fleisher, MD

Department of Anesthesiology and Critical Care Medicine, University of Pennsylvania School of Medicine, 3400 Spruce Street, Dulles Building, 6th floor, Philadelphia, PA 19104, USA

e-mail: elkassan@uphs.upenn.edu that the reported incidence of postoperative delirium ranges from 5.1 to $52.2 \% .^{3}$

As our society grows older, the impact of problems that are more prevalent in the elderly age group becomes more profound. Delirium was found to be an independent predictor of increased mortality, length of hospital stay, and postoperative complications. ${ }^{4}$ A recent study estimated the annual cost of this syndrome to be as much as 152 billion dollars, ${ }^{5}$ with an increased cost of approximately $\$ 2,500$ per hospital stay for each individual developing the syndrome. ${ }^{6}$

The pathophysiology of postoperative delirium is poorly understood. The stress responses to surgery and anesthesia, inadequate perfusion of the brain cells, and disturbance in metabolism, electrolytes, and neurotransmitters have been implicated as possible mechanisms for postoperative delirium. ${ }^{7}$ Because of the nature of the syndrome, animal models are problematic, and creating a study design that can point out a single cause for postoperative delirium is almost impossible. ${ }^{8}$ Our understanding of POD has been further confounded by a lack of well-designed randomized prospective clinical trials addressing a causal relationship between risk factors, including medications and POD.

The complexity of the syndrome calls for an organized team approach. Physicians, nurses, and family members should all be involved in the planned care of patients at risk. The team should begin by identifying patients at risk for POD when they present for the pre-admission screening process. Next, they should focus on modifying the risk factors that are modifiable. Drugs are the most common reversible causes of delirium. Anticholinergics, $\mathrm{H}_{2}$-blockers, benzodiazepines, opioids, and antipsychotic medications should be replaced with drugs that have no central effects.

The current study adds beta-blockers to the list of medications that may increase the probability of POD. Growing concern is being expressed about the potential 
side effects of perioperative beta-blockade administration. The POISE trial ${ }^{9}$ demonstrated an increased rate of stroke and death associated with a reduced incidence of non-fatal myocardial infarction in patients who were randomized to the beta-blockers. The pathophysiologic basis for a relationship between beta-blockade and ischemic cerebral insult is plausible, if unproven. The cerebral circulation is controlled by vasculature rich in beta-adrenergic receptors that mediate vasodilatation, and perfusion could be compromised to the detriment of cerebral metabolic functioning in individuals whose beta-receptors are blocked. Additionally, in individuals operating at the lower limit of their cerebral autoregulatory range, the central hemodynamic effects of beta-blockade might simply make cerebral perfusion inadequate. However, it is far less clear how beta-blockade could explain POD in individuals whose cerebral blood flow is not impaired.

Rather than increasing the probability of POD, some medications may actually lower the likelihood. Low-dose haloperidol did not show efficacy in reducing the incidence of postoperative delirium when compared with placebo in elderly hip surgery patients. However, the duration and severity of symptoms of delirium were less in the halopedirol group. ${ }^{10}$ Similarly, rivastigmine, a cholinesterase inhibitor, was ineffective in prophylaxis against POD after cardiac surgery. ${ }^{11}$ Statins have shown some protective effect against POD. ${ }^{12,13}$ The neuroprotective effect of statins can be attributed to its influence on inflammation and endothelial cell function. A non-pharmacological approach is equally important in prophylaxis against POD. A randomized trial demonstrated that proactive geriatrics consultation can reduce the incidence of delirium in elderly patients undergoing hip fracture repair. ${ }^{14}$

How should we apply the current study to clinical practice? Katznelson et al. utilized a retrospective chart review and standard logistic regression to determine the association between delirium and the use of perioperative medication. Such techniques tend to generate hypotheses, since there remains the potential for unmeasured confounders. Although the authors report the type and dose of each beta-blocker, they do not provide a dose-response curve for these agents to indicate whether a true relationship is a function of any perioperative beta-blocker or whether it is dose-related. Additional studies are required to confirm this association. However, the above discussion about the potential pathophysiologic link between these agents and perioperative delirium provides additional evidence that caution should be exercised in acutely starting these agents in patients at risk for delirium. Analogous to the risk of stroke (another adverse neurologic outcome) in patients, the risks of neurologic morbidity must be weighed against the risk of developing a myocardial event in patients who might benefit from a perioperative beta-blocker, and a preoperative assessment may be critical. Similarly, this study provides further support for continuing perioperative statin therapy in patients already on statins and additional support for initiating statins in high-risk patients.

In summary, the anesthesiologist needs to be cognizant of the risk of postoperative delirium in the elderly and strategies to reduce those risks. The main focus of intraoperative management should be on maintaining adequate ventilation, oxygenation, cerebral perfusion, and normal electrolytes and acid-base balance. Short-acting drugs are preferable in this setting. We believe that this study adds to the available literature. Most importantly, it suggests that further research is needed to determine the potential side-effects of chronic medications administered in the perioperative period and specifies the patient groups that are most at risk for manifesting those side effects.

\section{Des pensées claires concernant le délirium postopératoire et les médicaments périopératoires: faut-il se faire du souci?}

Le délirium est décrit depuis l'époque d'Hippocrate. En 1837, Sir Charles Dickens fait la description détaillée suivante de ce syndrome dans Les papiers posthumes $d u$ Pickwick Club - Histoire d'un clown, «Le ton de ce récit est également un mélange de bouffonnerie frivole et de terreur abjecte. Illusions et hallucinations sont foison $» .1$ Depuis, notre compréhension de cette maladie a évolué. En tant qu'anesthésiologistes et de concert avec les autres médecins impliqués dans les soins aux patients pendant la période périopératoire, nous nous faisons du souci quant au délirium postopératoire. Dans ce numéro du Journal, Katznelson et coll. ${ }^{2}$ ajoutent leurs écrits à la littérature sur ce sujet en réalisant une étude rétrospective afin de décrire l'association entre l'utilisation périopératoire de bêta-bloquants et de statines et la fréquence du délirium postopératoire chez les patients subissant une chirurgie vasculaire. Ils font état d'un doublement du rapport de cotes de délirium postopératoire chez les patients recevant des bêta-bloquants avant l'opération (rapport de cotes 2,06 avec un intervalle de confiance à $95 \%[1,18$ $3,6])$ et d'une réduction de $44 \%$ du rapport de cotes de délirium postopératoire chez les patients recevant des statines.

Le délirium est une voie terminale commune à plusieurs types de lésions, lesquelles mettent en péril un système nerveux affaibli et qui ne bénéficie que de réserves fonctionnelles limitées. D'un point de vue clinique, on le définit 
comme un état de confusion aigu caractérisé par la désorientation, un cycle de sommeil-éveil troublé, une détérioration de la mémoire, des troubles de la perception et une activité psychomotrice altérée. En raison de ces symptômes disparates et subjectifs, il n'est pas surprenant que l'incidence rapportée de délirium postopératoire aille de $5,1 \%$ à $52,2 \%{ }^{3}$

Avec le vieillissement de notre société, les répercussions de problèmes plus prévalents chez les personnes âgées deviennent plus profondes. Il a été démontré que le délirium est un prédicteur indépendant d'augmentation de la mortalité, de la durée de séjour à l'hôpital et des complications postopératoires. ${ }^{4}$ Une étude récente estimait que le coût annuel de ce syndrome se chiffrait à 152 milliards de dollars, ${ }^{5}$ avec un coût accru d'environ $2500 \$$ par séjour à l'hôpital pour chaque individu touché par ce syndrome. ${ }^{6}$

La physiopathologie du délirium postopératoire est peu comprise. Les réponses de stress à la chirurgie et à l'anesthésie, à une perfusion inadaptée des cellules du cerveau, et les troubles du métabolisme, des électrolytes et des neurotransmetteurs sont certains des mécanismes pouvant possiblement provoquer le délirium postopératoire. ${ }^{7}$ En raison de la nature de ce syndrome, les modèles animaux posent problème, et il est pratiquement impossible d'élaborer une méthodologie d'étude à même de déceler une cause unique au délirium postopératoire. ${ }^{8}$ Notre compréhension du délirium postopératoire a en outre été déjouée par le manque d'études cliniques prospectives randomisées bien conçues portant sur une relation causale entre les divers facteurs de risque, notamment les médicaments et le délirium postopératoire.

La complexité de ce syndrome nécessite une approche d'équipe organisée. Les médecins, les infirmières et les membres de la famille devraient tous être impliqués dans les soins prévus pour les patients à risque. L'équipe devrait d'abord identifier les patients exhibant un risque de délirium postopératoire lorsqu'ils se présentent à l'évaluation préopératoire avant l'admission. Ensuite, ils devraient se concentrer sur la modification des facteurs de risque qui sont modifiables. Les médicaments sont la cause réversible la plus courante de délirium. Les anticholinergiques, les antagonistes des récepteurs $\mathrm{H}_{2}$, les benzodiazépines, les opiacés et les médicaments antipsychotiques devraient être remplacés par des médicaments n'ayant pas d'effet sur le système nerveux central.

L'étude de Katznelson ajoute les bêta-bloquants à la liste des médicaments qui pourraient augmenter la probabilité d'un délirium postopératoire. Les effets secondaires potentiels d'une administration périopératoire de bêtabloquants sont une préoccupation croissante. L'étude POISE $^{9}$ a démontré un taux accru d'accident vasculaire cérébral et de décès associé à une incidence réduite d'infarctus du myocarde non mortel chez les patients randomisés à recevoir des bêta-bloquants. Le fondement physiopathologique d'une relation entre les bêta-bloquants et une lésion cérébrale ischémique est plausible, mais il demeure à prouver. La circulation cérébrale est contrôlée par une vasculature riche en récepteurs bêta-adrénergiques qui sont responsables de la vasodilatation, et la perfusion pourrait être compromise au détriment du fonctionnement métabolique cérébral chez les individus dont les bêtarécepteurs sont bloqués. De plus, chez les individus fonctionnant à la limite inférieure de leur capacité cérébrale d'autorégulation, les effets hémodynamiques centraux des bêta-bloquants pourraient simplement rendre la perfusion cérébrale inadaptée. Toutefois, il est bien plus difficile de comprendre comment les bêta-bloquants pourraient expliquer la présence d'un délirium postopératoire chez les individus dont le débit sanguin cérébral n'est pas affecté.

Plutôt que d'augmenter la probabilité de délirium postopératoire, certains médicaments pourraient en fait la diminuer. L'halopéridol à faible dose ne s'est pas montré efficace pour réduire l'incidence du délirium postopératoire par rapport à un placebo chez des patients âgés subissant une chirurgie de la hanche. Cependant, la durée et la sévérité des symptômes de délirium étaient moindres dans le groupe halopéridol. ${ }^{10}$ De la même façon, la rivastigmine, un inhibiteur de la cholinestérase, s'est avérée inefficace lorsqu'elle était administrée en prophylaxie contre le délirium postopératoire après une chirurgie cardiaque. ${ }^{11}$ Les statines ont démontré un certain effet protecteur contre le délirium postopératoire. ${ }^{12,13}$ L'effet neuroprotecteur des statines peut être attribué à leur impact sur l'inflammation et la fonction des cellules endothéliales. Une approche non pharmacologique est tout aussi importante pour prévenir le délirium postopératoire. Une étude randomisée a démontré qu'une consultation proactive en gériatrie pouvait réduire l'incidence de délirium chez les patients âgés subissant une chirurgie de réparation de la hanche. ${ }^{14}$

De quelle manière devrions-nous appliquer les résultats de l'étude présentée dans ce numéro à la pratique clinique? Katznelson et coll. ont eu recours à un modèle d'étude rétrospective des dossiers et à une analyse par régression logistique standard pour décrire l'association entre le délirium et l'utilisation de médicaments périopératoires. Une telle méthodologie a tendance à générer des hypothèses, étant donné que d'autres facteurs non mesurés pourraient également jouer un rôle. Bien que les auteurs rapportent le type et le dosage de chaque bêtabloquant, ils ne présentent cependant pas de courbe de dose-réponse pour ces agents. Une telle courbe permettrait d'indiquer si une relation véritable est une fonction de tout bêta-bloquant périopératoire ou si elle est liée à la dose. D'autres études sont nécessaires afin de confirmer 
cette association. Néanmoins, la discussion présentée cidessus au sujet du lien physiopathologique potentiel entre ces agents et le délirium périopératoire fournit des données probantes supplémentaires qui renforcent que la prudence est de mise lorsqu'on initie ces agents dans une phase aiguë chez des patients présentant un risque de délirium. Tout comme le risque d'accident vasculaire cérébral (un autre devenir neurologique néfaste) chez les patients, les risques de morbidité neurologique doivent être soupesés contre le risque de manifester une lésion myocardique chez les patients qui pourraient bénéficier d'un bêta-bloquant périopératoire, et une évaluation préopératoire pourrait s'avérer cruciale. De même, cette étude appuie également la continuation d'un traitement périopératoire à base de statines chez les patients déjà sous statines; elle fournit également un soutien supplémentaire à l'administration de statines chez des patients à haut risque.

En résumé, l'anesthésiologiste doit être conscient du risque de délirium postopératoire chez les patients âgés et connaître les stratégies à sa disposition pour réduire ces risques. La prise en charge peropératoire de soutien devrait se concentrer sur le maintien d'une ventilation, d'une oxygénation et d'une perfusion cérébrale adaptées, ainsi que sur des électrolytes normaux et un équilibre acidebase. Les médicaments à courte action sont préférables dans ce contexte. Nous pensons que cette étude ajoute de nouvelles pistes à la littérature existante à ce sujet. Mais surtout, elle soutient que des recherches supplémentaires sont nécessaires afin de définir les effets secondaires potentiels des médicaments chroniques administrés pendant la période périopératoire et spécifie les groupes de patients qui sont le plus à risque de manifester ces effets secondaires.

Competing interests None declared.

\section{References}

1. Dickens $C$. The Pickwick Papers. Available for free on Project Gutenberg; 1837

2. Katznelson R, Djaiani G, Mitsakakis $N$, et al. Delirium following vascular surgery: increased incidence with preoperative $\beta$-blocker administration. Can J Anesth 2009; 56. doi:10.1007/s12630-0099148-0

3. Dasgupta M, Dumbrell AC. Preoperative risk assessment for delirium after noncardiac surgery: a systematic review. J Am Geriatr Soc 2006; 54: 1578-89.

4. Cole MG. Delirium in elderly patients. Am J Geriatr Psychiatry 2004; 12: 7-21.

5. Leslie DL, Marcantonio ER, Zhang Y, Leo-Summers L, Inouye $S K$. One-year health care costs associated with delirium in the elderly population. Arch Intern Med 2008; 168: 27-32.

6. Inouye SK. Delirium in older persons. N Engl J Med 2006; 354: 1157-65.

7. Parikh SS, Chung F. Postoperative delirium in the elderly. Anesth Analg 1995; 80: 1223-32.

8. Inouye SK, Ferrucci L. Elucidating the pathophysiology of delirium and the interrelationship of delirium and dementia. J Gerontol A Biol Sci Med Sci 2006; 61: 1277-80.

9. Buksinska-Lisik M. Effects of extended-release meoprolol succinate in patients undergoing non-cardiac surgery (POISE trial): a randomised controlled trial-POISE Study Group (Polish). Kardiol Pol 2008; 66: 915-6; discussion 917-8.

10. Kalisvaart KJ, de Jonghe JF, Bogaards MJ, et al. Haloperidol prophylaxis for elderly hip-surgery patients at risk for delirium: a randomized placebo-controlled study. J Am Geriatr Soc 2005; 53 : 1658-66.

11. Gamberini M, Bolliger D, Lurati Buse GA, et al. Rivastigmine for the prevention of postoperative delirium in elderly patients undergoing elective cardiac surgery - a randomized controlled trial. Crit Care Med 2009; 37: 1762-8.

12. Katznelson R, Djaiani GN, Borger MA, et al. Preoperative use of statins is associated with reduced early delirium rates after cardiac surgery. Anesthesiology 2009; 110: 67-73.

13. Redelmeier DA, Thiruchelvam D, Daneman $N$. Delirium after elective surgery among elderly patients taking statins. CMAJ 2008; 179: 645-52.

14. Gustafson Y, Brannstrom B, Berggren D, et al. A geriatricanesthesiologic program to reduce acute confusional states in elderly patients treated for femoral neck fractures. J Am Geriatr Soc 1991; 39: 655-62. 